\title{
Ultrasound Guided Erector Spinae Block with Costotransverse Ligament Puncture Is More Effective than Erector Spinae Block Alone; Eight Cases for Oncologic Breast Surgery; A Brief Technical Report
}

\author{
Ayhan Şahin 1*, Ahmet Gültekin1, Ilker Yildirım1, Onur Baran², Cavidan Arar1 \\ ${ }^{1}$ Department of Anesthesiology and Reanimation, Medical Faculty of Namık Kemal University, Tekirdağ, Turkey \\ ${ }^{2}$ Clinic of Anesthesiology and Reanimation, Palandöken State Hospital, Erzurum, Turkey \\ Email: ^drayhan.sahin@hotmail.com, ahmetgultekin82@yahoo.com,drilkeryildirim@yahoo.com, \\ cavidanarar@yahoo.com,dronurbaran@hotmail.com
}

How to cite this paper: Şahin, A., Gültekin, A., Yildirım, I., Baran, O. and Arar, C. (2020) Ultrasound Guided Erector Spinae Block with Costotransverse Ligament Puncture Is More Effective than Erector Spinae Block Alone; Eight Cases for Oncologic Breast Surgery; A Brief Technical Report. Open Journal of Anesthesiology, 10, 179-189. https://doi.org/10.4236/ojanes.2020.105016

Received: April 10, 2020

Accepted: May 11, 2020

Published: May 14, 2020

Copyright $\odot 2020$ by author(s) and Scientific Research Publishing Inc. This work is licensed under the Creative Commons Attribution International License (CC BY 4.0).

http://creativecommons.org/licenses/by/4.0/

\begin{abstract}
Objectives: Due to the complex breast innervation, postoperative analgesia after breast surgery is a challenge for the anesthesiologists. The erector spinae plane block (ESP) is a newly defined promising technique for this purpose. Since the description by Forero, ultrasound-guided erector spinae block (ESP) has performed for several surgeries for postoperative analgesia. Many regional methods pectoral nerve block (PECs), serratus plane block (SPB), were described in the literature for maintaining postoperative analgesia. Among all these regional anesthesia techniques, paravertebral block (PVB) is the most studied and found to be a valid regional technique for this purpose. Due to its anatomic proximity to the pleura and central neuraxial system, it's also one of the most challenging techniques. Materials and Methods: Patients aged between 37 and 47 years, American Society of Anesthesiologists physical status I-II and scheduled for elective unilateral modified radical mastectomy (MRM) surgery without axillary lymph node dissection were enrolled to this case series. In this report, we describe a novel combination of ESP and simplified PVB block and its successful application in 8 cases of oncologic breast surgery. In our clinic, ESP block is performed for all oncological breast cases. Eight cases of MRM without axillary dissection were randomly selected by the

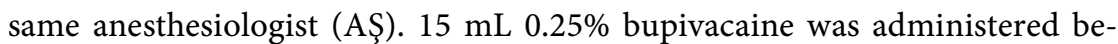
tween the erector spinae muscles and the transverse process at the level of the 4th thoracal vertebra with the caudo-cranial approach. Additionally, the
\end{abstract}


needle tip was directed to T4 superior costotransverse ligament, and the ligament was perforated at the top point where it attaches to the T4 transverse process. Results: The ESP block is used for a local anesthetic depot, as a safe and straightforward technique and costotransverse ligament puncture facilitates diffusion and ensures local anesthetic passage to the paravertebral area for thoracic postsurgical analgesia. Using lower doses, comparing this combination with all studies with ESP, it appears that there are a better Numeric Pain Rating Scale (NPRS) scores in the first postoperative hours. NPRS scores at the 12th and 24th hours appear to be similar to those performed in ESP without costotransverse ligament puncture. Conclusion: We submit the first description of a novel combination of ESP block and simplified paravertebral block (PVB) and its successful application in 8 cases of oncologic breast surgery.

\section{Keywords}

Erector Spinae Block, Costotransverse Ligament, Paravertebral Block, Breast Surgery, Oncology

\section{Introduction}

Many regional anesthesia techniques have been described for post-breast surgery analgesia [1]. Paravertebral block (PVB) is the most effective experienced technique, but it is compelling due to adjacent to pleura and central neuraxial system [2]. Erector spinae block (ESP), a comparatively safe and straightforward technique, has been used for postoperative analgesia in breast surgeries [3] [4] [5].

The ESP block is performed by depositing the local anesthetic (L.A.) in the fascial plane, more profound than the erector spinae muscle (ESM) at the tip of the transverse process of the vertebra. Therefore, LA is distributed in the craniocaudal fascial plane, one dermatome, a median of each $3.4 \mathrm{ml}$ of injected volume [6]. Additionally, it diffuses anteriorly to the paravertebral and epidural spaces and laterally to the intercostal space at several levels [7]. Furthermore, the diffusion of L.A to the paravertebral space through the costotransverse foramina and the intertransverse complex (intertransverse and costotransverse ligaments: levators, rotators, and intercostal muscles) provides both visceral and somatic analgesia. This diffusion into the epidural space and the neural foramina has been reported in anatomical studies that examined 2 to 5 vertebral levels that were centered near the injection site. However, this diffusion was not observed consistently in all cadaver studies. We claimed that ESP blocks with cost transverse ligament puncture would ensure more effective pain control and fewer onset times of ESP blocks according to ESP blocks alone.

We present the first description of a novel combination of ESP block and simplified PVB, due to the mechanism of ESP block and its successful application in 8 cases of oncologic breast surgery. We discuss the onset times and numeric pain 
rating scales (NPRS) ranging from 0 (no pain) to 10 (worst imaginable pain), which is used for postoperative pain assessment.

\section{Methods}

\section{ESP with Costotransverse Ligament Puncture Technique}

Written informed consent was provided by all patients for inclusion in this report. Patients aged between 37 and 47 years, American Society of Anesthesiologists physical status I-II and scheduled for elective unilateral modified radical mastectomy surgery (ASA) without axillary lymph node dissection were enrolled in this report. The exclusion criteria were patients with coagulation disorder, chronic opioid use, hepatic or renal insufficiency, local infection at the injection site, spine or chest wall deformity, known allergy to local anesthetics. Blocks were performed $30 \mathrm{~min}$ before the induction of general anesthesia in the regional anesthesia block room. Patients were given 4 - $5 \mathrm{lt} / \mathrm{O}_{2}$ and underwent routine monitorization, including electrocardiogram, peripheral oxygen saturation, and non-invasive blood pressure. Intravenous access was secured, and sedation with midazolam $2 \mathrm{mg}$ was administered.

Blocks were performed in the sitting position, and $10 \%$ povidone-iodine was used for skin disinfection. Blocks were performed with the same anesthesiologist (AŞ). Linear, high-frequency probe $(8-19 \mathrm{MHz})$ of Esaote My Lab 6 U.S. machine (Florence, Italy) with a $22 \mathrm{G}, 50 \mathrm{~mm}$, insulated facet type needle (BBraun Sonoplex, Melsungen, Germany) were used for blocks. Blocks were performed in an in-plane technique at level T4 caudo-cranial direction.

The transverse process and the superior costotransverse ligament are important sonoanatomical landmarks for this technique using sagittal transducer guidance. The probe was placed $2-3 \mathrm{~cm}$ lateral to the midline with the sagittal approach. Three muscles were identified superficial to the hyperechoic transverse process shadow as follows: trapezius, rhomboid major, and erector spinae. After the costotransverse ligament and T4 transverse process were captured on the same ultrasound image, then a 22-gauge, $50 \mathrm{~mm}$ needle was first directed to the T4 transverse process. The correct position of the needle tip was confirmed with the administration of $0.5-1 \mathrm{ml}$ of local anesthetic (L.A.), and then $15 \mathrm{ml}$ of $0.25 \%$ bupivacaine was administered (Figure 1). Both cranial and caudal L.A. spread was observed. Then, the needle tip was directed to T4 superior costotransverse ligament cranially, and the ligament was perforated at the top point where it attaches to the T4 transverse process. After perforating the costotransverse ligament, no drug was injected, and no aspiration was made, then the needle was pulled back out of the skin (Figure 2).

Each patient received $15 \mathrm{ml}$ of $0.25 \%$ bupivacaine. The success of the block and extension of sensory loss was evaluated using the pinprick test began just 10 min after the injection of local anesthetic. The block is accepted as a failed block if the loss of sensation was not attained within each patient received $15 \mathrm{ml}$ of $0.25 \%$ bupivacaine. The success of the block and extension of sensory loss were 


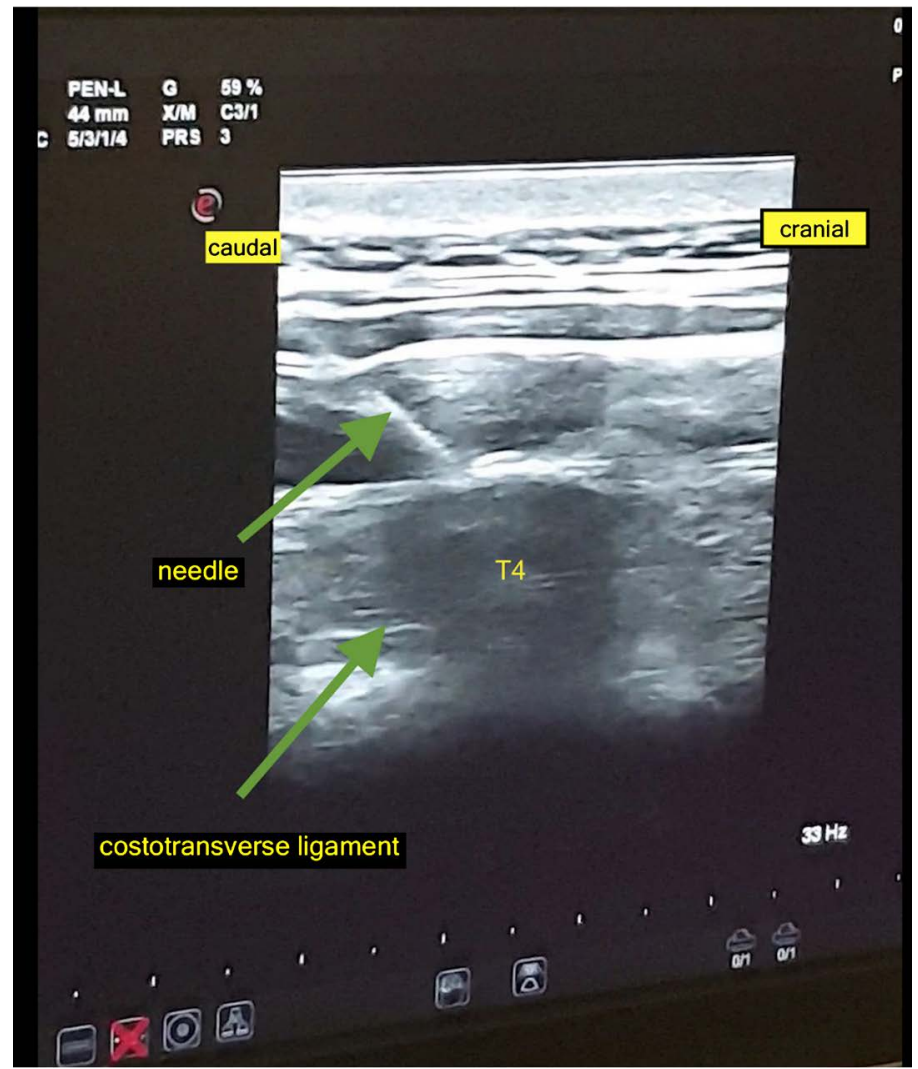

Figure 1. Ultrasound image showing the caudo-cranial approach and surrounding anatomic landmarks. ESP block performed at the T4 level. Cranial to caudal approach. Local anesthetic spread and needle position. Costotransverse ligament where it adheres to the T4 transverse process.

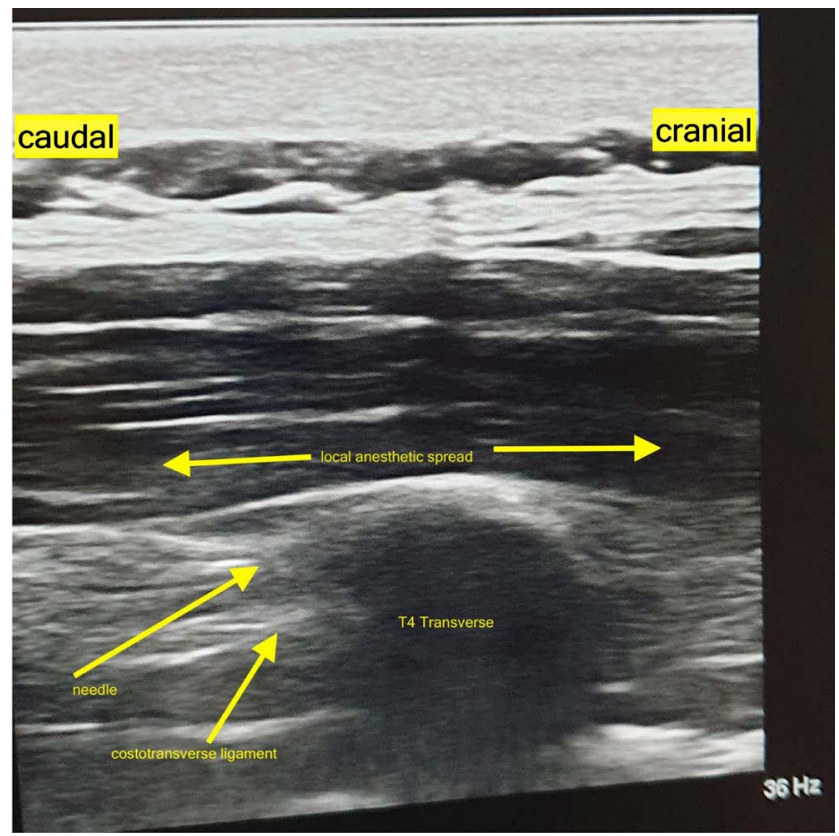

Figure 2. Ultrasound image illustrating costotransverse ligament puncture at T4 level after ESP block. Cranial to the caudal position. Needle tip pierces the top of the costotransverse ligament-local anesthetic under the erector spinae muscle as a depot. 
evaluated using the pinprick test began just 10 min after the injection of local anesthetic. The block is accepted as a failed block if the loss of sensation was not attained within $30 \mathrm{~min}$.

\section{Anesthesia Protocol}

The same G.A. technique was used for all patients. Basic monitoring was done (adding capnography to previous monitoring techniques). Intravenous induction was done using fentanyl $1 \mu \mathrm{g} / \mathrm{kg}$, propofol $2 \mathrm{mg} / \mathrm{kg}$ and Rocuronium 0.6 $\mathrm{mg} / \mathrm{kg}$. Anesthesia was maintained by administering sevoflurane $1.5 \%-2 \%$ in a mixture of oxygen and air and Rocuronium $0.1 \mathrm{mg} / \mathrm{kg}$ IV as required. The ventilator settings were adjusted to keep EtCO2 between 35 and $40 \mathrm{mmHg}$. A combination of sevoflurane with air in oxygen (1:1) in $3 \mathrm{~L}$ of fresh gas flow was used for anesthesia maintenance. At the end of the surgery, paracetamol $1 \mathrm{~g}, 100 \mathrm{mg}$ iv was administered for providing postoperative analgesia, and also, Ondansetron $8 \mathrm{mg}$ was given for the prevention of postoperative nausea and vomiting.

An anesthesiologist (AG) who was blinded to the costotransverse puncture has recorded NPRS scores at postoperative 1, 4, 8, 12, and $24 \mathrm{~h}$. The same anesthesiologist also recorded total tramadol consumptions during the $24 \mathrm{~h}$ postoperative period and the incidence of postoperative nausea and vomiting (PONV).

\section{Results}

Demographical data, durations were similar, and types of surgeries were the same (Table 1). Since all of the blocks were performed at the T4 level, cases whose axillary dissection was planned from the beginning of surgery or turned

Table 1. NPRS: Numeric Pain Rating Scale ranges from 0 (no pain) to 10 (worst pain imaginable); ASA 1 and 2: American Society of Anesthesiologists Scores; PONV: postoperative nausea and vomiting; MRM: modified radical mastectomy.

\begin{tabular}{|c|c|c|c|c|c|c|c|c|}
\hline & Case 1 & Case 2 & Case 3 & Case 4 & Case 5 & Case 6 & Case 7 & Case 8 \\
\hline \multirow{3}{*}{$\begin{array}{c}\text { Demographic/clinical } \\
\text { details }\end{array}$} & 42-year-old & 37-year-old & 38-year-old & 52-year-old & 30-year-old & 38-year-old & 47-year-old & 37-year-old \\
\hline & $157 \mathrm{~cm} 76 \mathrm{~kg}$ & $170 \mathrm{~cm} 73 \mathrm{~kg}$ & $160 \mathrm{~cm} 58 \mathrm{~kg}$ & $170 \mathrm{~cm} 108$ & $162 \mathrm{~cm} 52 \mathrm{~kg}$ & $159 \mathrm{~cm} 58 \mathrm{~kg}$ & $155 \mathrm{~cm} 69 \mathrm{~kg}$ & $160 \mathrm{~cm} 77 \mathrm{~kg}$ \\
\hline & ASA 2 & ASA 1 & ASA 1 & kg ASA 2 & ASA 1 & ASA 1 & ASA 2 & ASA 1 \\
\hline Surgery type & MRM & MRM & MRM & MRM & MRM & MRM & MRM & MRM \\
\hline Gender & Female & Female & Female & Female & Female & Female & Female & Female \\
\hline Duration of surgery & 2 hours & 2.5 hours & 2 hours & 2.5 hours & 3 hours & 2 hours & 2.5 hours & 2.5 hours \\
\hline NPRS postop 1 hour & 1 & 2 & 0 & 1 & 1 & 0 & 1 & 2 \\
\hline NPRS postop 4 hours & 0 & 1 & 0 & 1 & 0 & 0 & 1 & 1 \\
\hline NPRS postop 8 hours & 0 & 1 & 0 & 2 & 0 & 1 & 1 & 1 \\
\hline NPRS postop 12 hours & 1 & 1 & 1 & 2 & 1 & 2 & 3 & 1 \\
\hline NPRS postop 24 hours & 2 & 3 & 2 & 2 & 2 & 3 & 3 & 2 \\
\hline $\begin{array}{l}\text { Postoperative total } \\
\text { tramadol consumption }\end{array}$ & None & $70 \mathrm{mg}$ & None & $100 \mathrm{mg}$ & $50 \mathrm{mg}$ & $50 \mathrm{mg}$ & $70 \mathrm{mg}$ & $80 \mathrm{mg}$ \\
\hline PONV & None & None & None & None & None & None & None & None \\
\hline Block onset time & $13 \mathrm{~min}$ & $11 \mathrm{~min}$ & $8.5 \mathrm{~min}$ & $15 \mathrm{~min}$ & $9 \min$ & $10 \mathrm{~min}$ & $13 \mathrm{~min}$ & $12 \mathrm{~min}$ \\
\hline
\end{tabular}


to axillary dissection according to the frozen result were excluded. No complications were observed in any case. The studies, comparing ESP block and PVB block before were examined, PVB was observed to be more effective [8]. In our new combination, NPRS scores were obtained close to PVB. Nausea and vomiting were not observed in any patient. Two of the patients had no postoperative Tramadol consumption. NPRS scores and postoperative total tramadol consumptions are better than ESP block alone according to the studies done before [9].

Comparing this combination with all studies performed only with ESP, it is seen that there are better NPRS scores in the first postoperative hours. The NPRS scores at the 12th and 24th hours seem to be similar to those performed in ESP without costotransverse ligament puncture.

\section{Discussion}

Although ESP blocks provide simpler and safer regional anesthesia techniques than traditional paravertebral blocks [10], the optimal injectate volume required for the successful single or multilevel paravertebral spread is unknown. A $20 \mathrm{ml}$ volume of injectate in single ESP block seems to be inadequate in clinical practice, in cadaveric studies analyzing injectate spread patterns in ESP blocks also used $20 \mathrm{ml}$ volume of dye correlatively [11] [12]. A recent cadaveric study [13] illustrated an exciting finding that there is no paravertebral spread in any $10 \mathrm{ml}$ performed ESP blocks. Furthermore, this study demonstrated with an endoscopic evaluation that using a $30 \mathrm{ml}$ dye solution, stained thoracic spinal nerves at the intervertebral foramina, and epidural spread was observed. Despite postmortem changes in the integrity of tissues that could affect the diffusion of the injectate, it seems paravertebral spread that occurs after ESP blocks is volume dependent. Chest movements during inspiration and expiration can steer the injectate solution to paravertebral or intercostal spaces.

Precisely at this point, this novel combination of ESP block and simplified PVB may be essential. First of all, interfascial blocks seem to be relatively safe from systemic toxicity associated with local anesthetics due to the low vascularity of fascial structures [14]. However, the potential for local anesthetic systemic toxicity was reported when performing ESP blocks with more than $40 \mathrm{ml}$ of diluted local anesthetics [15]. This combination may be necessary for patients undergoing bilateral thoracal ESP to avoid toxicity, or it may be suitable for physiological conditions with local anesthetic susceptibility such as pregnancy, liver failure. Using this combination may lead to an adequate effect with lower doses and volumes.

We tried to describe this technique by combining ESP and facilitated PVB with this series of case reports. The sample size may be small but sufficient to explain how the technique is and how effective it is. This technical report demonstrated that the combination of ESP block and costotransverse ligament puncture is more effective with lower doses of local anesthetics. We also dem- 
onstrated that NPRS scores in the first postoperative hours. Therefore, costotransverse ligament acts as a barrier for ESP blocks clinically.

However, as the paravertebral space (PVS) is often small, even with the guidance of ultrasound, the risk for a pleural puncture, especially with a less skilled anesthesiologist, is still present. With this combination, due to the needle tip time to stay under the costotransverse ligament is very short and no negative aspiration is not required, it seems to be a practical method. Moreover, since no local anesthetic is given to the paravertebral area, no volume-related complications seen. None of the complications, such as nausea, vomiting, hypotension seen in the paravertebral block, observed with this combination.

The PVS is filled with fat and is traversed by the dorsal branches and ventral branches of spinal nerves, communicating branches, intercostal nerves and blood vessels, hemiazygos vein, thoracic duct and sympathetic trunk [16]. Placing a catheter or making injection in a small area with so many structures is highly associated with complications. In our technique, we are drilling through the costotransverse ligament at the top point where it attaches and drawback without going deep into the paravertebral area, and not keeping the needle tip for a short time protects all these structures and thus avoid complications.

In studies comparing PVB with ESP, the complication rate was higher in the PVB group [17]. The most important benefits of this new method are the absence of bilateral block, hypotension, intrathecal spread from PVB complications. The risk of pneumothorax is still constant but reduced.

Conventionally, the superior costotransverse ligament was assumed to be a compact ligament and that an effective PVB could only be achieved after direct penetration of the ligament. However, the PVB and intercostal nerves could be stained even if the dye was injected above the superior costotransverse ligament or intercostal muscle through the porous tissue around the superior costotransverse ligament [18]. Another study reported gaps between the medial and lateral portions of the superior costotransverse ligament [19]. Similar dye spreading patterns to the PVB were also reported in retrolaminar blocks, another alternative to paravertebral blocks, without pleural displacement or superior costotransverse ligament penetration [20].

Despite the fact that, the mechanism of the ESP remains unclear, local anesthetics injected beneath the erector spinae muscle (interfacial injection) can spread through the costotransverse foramen and peripheral porous tissues into the intercostal and thoracic paravertebral spaces [21] [22]. The costotransverse foramen is a bony gap between the neck of a rib and the TP of a vertebra. A cadaveric study reported that tracer dyes injected beneath the erector spinae muscle can spread into the deep structures of the external intercostal muscle and internal intercostal membrane through the costotransverse foramen [21]. We estimate that, this technique creates a tunnel between the paravertebral area and the ESP block, which acts as a local anesthetic depot. Reduced paresthesia onset times and better NPRS scores than ESP block during the first hours of the post- 
operative period supports this.

The ultrasound-guided retrolaminar block (RLB) is performed by ultrasound imaging in a paramedian sagittal plane by sequentially visualizing the pleura and ribs, transverse processes, and the corresponding laminae (from lateral to medial). The block needle is guided to contact the lamina, and the local anesthetic injectate is visualized under real-time imaging [23]. The actual distribution of the injectate with the ultrasound-guided retrolaminar block is not known and will be the subject of further studies. These studies will most likely rely on direct anatomic investigation, as clinical studies that rely on dermatomal mapping after paravertebral blockade are unable to distinguish among local anesthesia in the paravertebral, intercostal, or epidural spaces. Yang et al. demonstrated that the injectates of RLB and ESP reached the paravertebral space and infiltrated the superior costotransverse ligament in unembalmed cadavers [24]. The area stained with dye in ESP was more lateral, whereas the dye spread vertically along the posterior surface of the lamina in RLB. These findings indicated that RLB could involve the dorsal rami of the spinal nerve and may be more suitable for the analgesia of the thoracic back region than is ESP. A recent anatomical study of RLB using porcine cadavers suggested that the dye was distributed to the paravertebral space in a volume-dependent manner [25]. Conventionally, RLB is also performed with $20 \mathrm{~mL}$ or higher volumes.

The major advantage of this technique, if achieved correctly, is minimizing or even eliminating the risk of pneumothorax. Additionally, the risks of nerve root damage and inadvertent injection into a dural sleeve, an intervertebral foramen, or the epidural or intrathecal spaces should also be decreased. Furthermore, this technique requires less skill, as the exact identification of the costotransverse ligament, it is not compelling in experienced hands.

\section{Limitations}

In the application of this technique, the practitioner must master both methods (ESP and PVB) in the thoracic region. It may not always be possible to display costotransverse ligament in obese patients and low-resolution ultrasound devices. The practitioner must have advanced ultrasound techniques knowledge. Because PVB-induced pneumothorax, pleural puncture, hematoma, paravertebral muscle pain complications still exist.

\section{Conclusion}

To the best of our knowledge, according to the published data suggest using 20 $\mathrm{mL}, 25 \mathrm{~mL}$, or higher volumes of local anesthetics of unilateral ESP block. The use of variable local anesthetics doses, often at the upper dose of limit, however, leaves question marks regarding the consistency and safety of the ESP block. Especially in bilateral blocks, since local anesthetics reach the toxic limit, blocking to another area goes beyond safety limits. For example, in patients undergoing mammoplasty and abdominoplasty, doing ESP block and Transversus abdominis 
plane (TAP) block simultaneously pushes the toxic dose limits.

Shorter block onset times also seem to be an advantage, thus reducing the need for intraoperative and postoperative analgesics. This new technique provides comfort to the patient and the practitioner in order to avoid the toxicity of local anesthetics and short onset times in bilateral breast surgeries. Also, this new technique seems to be suitable for postoperative analgesia for multiple operations performed simultaneously. Postoperative analgesia for breast reconstruction with autologous tissue following mastectomy operations can be very convenient with this technique. Latissumus dorsi myocutaneous flap (LDMF), transverse rectus abdominis myocutaneous flap (TRAM), Fat grafting from the abdominal wall are used for his operations, and with this new technique, TAP block can be added by avoiding local anesthetic toxicity.

\section{Conflicts of Interest}

The authors declare no conflicts of interest regarding the publication of this paper.

\section{References}

[1] Hong, B., Bang, S., Chung, W., et al. (2019) Multimodal Analgesia with Multiple Intermittent Doses of Erector Spinae Plane Block through a Catheter after Total Mastectomy: A Retrospective Observational Study. The Korean Journal of Pain, 32, 206-314. https://doi.org/10.3344/kjp.2019.32.3.206

[2] Talawar, P., Kumar, A., Bhoi, D., et al. (2019) Initial Experience of Erector Spinae Plane Block in Patients Undergoing Breast Surgery: A Case Series. Saudi Journal of Anaesthesia, 13, 72-74.

[3] Selvi, O. and Tulgar, S. (2019) Use of the Ultrasound-Guided Erector Spinae Plane Block in a Segmental Mastectomy. Turkish Journal of Anaesthesiology \& Reanimation, 47, 158-160. https://doi.org/10.5152/TJAR.2019.50024

[4] Altıparmak, B., Toker, M.K., Uysal, A.İ., et al. (2019) Comparison of the Efficacy of Erector Spinae Plane Block Performed with Different Concentrations of Bupivacaine on Postoperative Analgesia after Mastectomy Surgery: Randomized, Prospective, Double-Blinded Trial. BMC Anesthesiology, 19, 31. https://doi.org/10.1186/s12871-019-0700-3

[5] Singh, S. and Chowdhary, N.K. (2018) The Erector Spinae Plane Blocks an Effective Block for Postoperative Analgesia in Modified Radical Mastectomy. Indian Journal of Anaesthesia, 62, 148-150. https://doi.org/10.4103/ija.IJA_726_17

[6] De Cassai, A. and Tonetti, T. (2018) Local Anesthetic Spread during Erector Spinae Plane Block. Journal of Clinical Anesthesia, 48, 60-61. https://doi.org/10.1016/j.jclinane.2018.05.003

[7] Vidal, E., Giménez, H., Forero, M., et al. (2018) Erector Spinae Plane Block: A Cadaver Study to Determine Its Mechanism of Action. Revista Española de Anestesiología y Reanimación, 65, 514-519. https://doi.org/10.1016/j.redar.2018.07.004

[8] El Ghamry, M.R. and Fawzy Amer, A. (2019) Role of Erector Spinae Plane Block versus Paravertebral Block in Pain Control after Modified Radical Mastectomy; A Prospective Randomized Trial. Indian Journal of Anaesthesia, 63, 1008-1014. https://doi.org/10.4103/ija.IJA_310_19

[9] Aksu, C., Kuş, A., Yörükoğlu, H.U., et al. (2019) Analgesic Effect of the Bi-Level Injection Erector Spinae Plane Block after Breast Surgery: A Randomized Controlled 
Trial. Agri, 31, 132-137. https://doi.org/10.14744/agri.2019.61687

[10] El-Boghdadly, K. and Pawa, A. (2017) The Erector Spinae Plane Block: Plane and Simple. Anesthesia, 72, 434-438. https://doi.org/10.1111/anae.13830

[11] Adhikary, S.D., Bernard, S., Lopez, H., et al. (2018) Erector Spinae Plane Block versus Retrolaminar Block: A Magnetic Resonance Imaging and Anatomical Study. Regional Anesthesia \& Pain Medicine, 43, 756-762. https://doi.org/10.1097/AAP.0000000000000798

[12] Yang, H.M., Choi, Y.J., Kwon, H.J., et al. (2018) Comparison of Injectate Spread and Nerve Involvement between Retrolaminar and Erector Spinae Plane Blocks in the Thoracic Region: A Cadaveric Study. Anesthesia, 73, 1244-1250.

https://doi.org/10.1111/anae.14408

[13] Choi, Y.-J., Kwon, H.-J., Jehoon, O., et al. (2019) Influence of Injectate Volume on the Paravertebral Spread in Erector Spinae Plane Block: An Endoscopic and Anatomical Evaluation. PLOS ONE, 14, e0224487. https://doi.org/10.1371/journal.pone.0224487

[14] Rahiri, J., Tuhoe, J., Svirskis, D., et al. (2017) A Systematic Review of the Systemic Concentrations of Local Anesthetic after Transversus Abdominis Plane Block and Rectus Sheath Block. British Journal of Anaesthesia, 118, 517-526. https://doi.org/10.1093/bja/aex005

[15] Luftig, J., Mantuani, D., Herring, A.A., et al. (2018) Successful Emergency Pain Control for Posterior Rib Fractures with Ultrasound-Guided Erector Spinae Plane Block. The American Journal of Emergency Medicine, 36, 1391-1396. https://doi.org/10.1016/j.ajem.2017.12.060

[16] Karmakar, M.K. (2001) Thoracic Paravertebral Block. Anesthesiology, 95, 771-780. https://doi.org/10.1097/00000542-200109000-00033

[17] Naja, Z. and Lönnqvist, P.A. (2001) Somatic Paravertebral Nerve Blockade Incidence of Failed Block and Complications. Anesthesia, 56, 1181-1201. https://doi.org/10.1046/j.1365-2044.2001.02084-2.x

[18] Costache, I., de Neumann, L., Ramnanan, C.J., Goodwin, S.L., Pawa, A., Abdallah, F.W., et al. (2017) The Mid-Point Transverse Process to Pleura (MTP) Block: A New End-Point for Thoracic Paravertebral Block. Anaesthesia, 72, 1230-1236. https://doi.org/10.1111/anae.14004

[19] Luyet, C., Eichenberger, U., Greif, R., Vogt, A., Szücs Farkas, Z. and Moriggl, B. (2009) Ultrasound-Guided Paravertebral Puncture and Placement of Catheters in Human Cadavers: An Imaging Study. British Journal of Anaesthesia, 102, 534-539. https://doi.org/10.1093/bja/aep015

[20] Damjanovska, M., Stopar Pintaric, T., Cvetko, E. and Vlassakov, K. (2018) The Ultrasound-Guided Retrolaminar Block: Volume-Dependent Injectate Distribution. Journal of Pain Research, 11, 293-299. https://doi.org/10.2147/JPR.S153660

[21] Forero, M., Adhikary, S.D., Lopez, H., Tsui, C. and Chin, K.J. (2016) The Erector Spinae Plane Block: A Novel Analgesic Technique in Thoracic Neuropathic Pain. Regional Anesthesia \& Pain Medicine, 41, 621-627. https://doi.org/10.1097/AAP.0000000000000451

[22] Adhikary, S.D., Bernard, S., Lopez, H. and Chin, K.J. (2018) Erector Spinae Plane Block versus Retrolaminar Block: A Magnetic Resonance Imaging and Anatomical Study. Regional Anesthesia \& Pain Medicine, 43, 756-762. https://doi.org/10.1097/AAP.0000000000000798

[23] Pfeiffer, G., Oppitz, N., Schone, S., Richter-Heine, I., Hohne, M. and Koltermann, C. (2006) Analgesia of the Axilla Using a Paravertebral Catheter in the Lamina 
Technique (German). Anaesthesist, 55, 423-427.

https://doi.org/10.1007/s00101-005-0969-0

[24] Yang H., Choi, Y.J., Kwon, H., Cho, T.H. and Kim, S.H. (2018) Comparison of Injective Spread and Nerve Involvement between Retrolaminar and Erector Spinae Plane Blocks in the Thoracic Region: A Cadaveric Study. Anaesthesia, 73, 1244-1250. https://doi.org/10.1111/anae.14408

[25] Damjanovska, M., Stopar Pintaric, T., Cvetko, E. and Vlassakov, K. (2018) The Ultrasound-Guided Retrolaminar Block: Volume-Dependent Injectate Distribution. Journal of Pain Research, 11, 293-299. https://doi.org/10.2147/JPR.S153660 